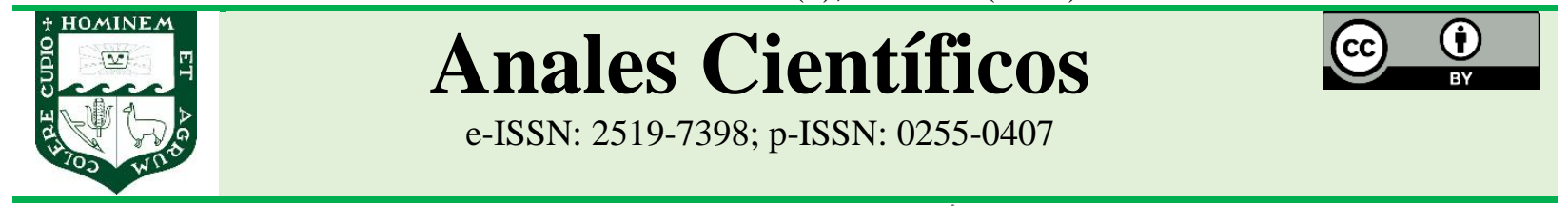

ARTÍCULO ORIGINAL - RESEARCH ARTICLE

http://dx.doi.org/10.21704/ac.v82i2.1764

\title{
EFECTOS DEL CIPIONATO Y BENZOATO DE ESTRADIOL EN VAQUILLAS MESTIZAS SOBRE EL PORCENTAJE DE PREÑEZ
}

\section{Effects of cypionate and estradiol benzoate in cross breed heifers on pregnancy rate}

\author{
Hjalmar Vera Alava ${ }^{1}$; Rodolfo Pedroso Sosa ${ }^{2}$; Gustavo Hidalgo Bravo ${ }^{3 *}$ (D) \\ ${ }^{1}$ Ministerio de Ganadería y Agricultura, Ecuador. \\ ${ }^{2}$ Instituto de Investigaciones, Facultad de Ciencias Veterinarias, Universidad Técnica de Manabí. Santa Ana, Manabí, \\ Ecuador. \\ ${ }^{3}$ Instituto Superior Tecnológico Enrique Noboa Arízaga, Carrera de Producción Agropecuaria. La Troncal Cañar. Ecuador. \\ *E-mail: turco1987@ hotmail.com
}

Recibido: 20/06/2020; Aceptado: 02/10/2021; Publicado: 31/12/2021

\begin{abstract}
The objective of this research was to determine the effect of Estradiol Cypionate (ECP), at the time of withdrawal of the Progesterone vaginal device or Estradiol Benzoate (EB), 24 hours later on the pregnancy rate in heifers inseminated at fixed time (FTAI). Seventy-two Bos indicus x Bos taurus crossbred heifers of body condition 3.0 to 3.5 (Scale 1 to 5), body weight between 300 to $400 \mathrm{~kg}$ were selected. The animals were divided into three groups A, B and C. All animals were synchronized to estrus using EB + Progesterone (DIB 0.5) + Prostaglandin F2-Alpha. Ovulation was induced by administering 0.5 and 1 milligram estradiol cypionate to groups A and B on day zero when the progesterone vaginal device was removed. To group C, 1 milligram of Estradiol Benzoate was administered 24 hours after the end of progesterone treatment. The administration of ECP at a dose of 0.5 milligram, showed the best uniformity in the manifestation of estrus, higher percentage of conception $68.1 \%$ and pregnancy $62.5 \%$ than the rest of the treated groups, with respect to body weight did not influence pregnancy. It is concluded that the administration of low dose ECP at zero hours after the end of the progesterone treatment showed better indicators on pregnancy percentages as well as allowing a one-day reduction in field work with adequate pregnancy rates. However, the range of 300 to $400 \mathrm{~kg}$ of body weight at the beginning of treatment had no effect on fertility in Bos indicus x Bos taurus crossbred heifers.
\end{abstract}

Keywords. Estradiol salts | tropical climate | crossbred heifers | ovulation.

\section{RESUMEN}

El objetivo de esta investigación fue determinar el efecto del Cipionato de Estradiol (ECP), al momento del retiro del dispositivo vaginal de Progesterona o el Benzoato de Estradiol (BE), 24 horas más tarde sobre la tasa de preñez en vaquillas inseminadas a tiempo fijo (IATF). Se seleccionaron 72 vaquillas mestizas Bos indicus x Bos taurus de condición corporal 3,0 a 3,5 (Escala 1 al 5), peso corporal entre 300 a 400 kilos. Los animales fueron divididos en 
Vera, H., Pedroso, R., \& Hidalgo, G. (2021). Anales Científicos. 82(2), 198-205. DOI. 10.21704/ac.v82i2.1764

tres grupos A, B y C, en todos los animales se les sincronizó el estro mediante el uso de BE + Progesterona (DIB $0.5)$ + Prostaglandina F2-Alfa. Se indujo ovulación administrando a los grupos A y B, Cipionato de Estradiol 0,5 y 1 miligramo, el día cero al retirar el dispositivo vaginal de progesterona. Al grupo $\mathrm{C}$, se administró 1 miligramo de Benzoato de Estradiol a las 24 horas de finalizado el tratamiento con progesterona. La administración de ECP en dosis 0,5 miligramo, mostró la mejor uniformidad en la manifestación del celo, mayor porcentaje de concepción $68,1 \%$ y preñez $62,5 \%$ que el resto de los grupos tratados, con respecto al peso corporal no influyo sobre la preñez. Se concluye que administrar ECP en dosis baja a las cero horas de finalizado el tratamiento con progesterona mostró unos mejores indicadores sobre los porcentajes de preñez como también permite disminuir un día en las labores de campo con adecuadas tasas de preñez, no obstante, el rango de 300 a $400 \mathrm{~kg}$ de peso corporal al inicio de tratamiento no tuvo efecto en la fertilidad en vaquillas mestizas Bos indicus x Bos taurus.

Palabras clave: Sales de estradiol | clima tropical | vaquillas mestizas | ovulación.

\section{Forma de citar el artículo (Formato APA):}

Vera, H., Pedroso, R., \& Hidalgo, G. (2021). Efectos del cipionato y benzoato de estradiol en vaquillas mestizas sobre porcentajes de preñez. Anales Científicos. 81(2), 198-205. http://dx.doi.org/10.21704/ac.v82i2.1764

Autor de correspondencia (*): Gustavo Hidalgo Bravo. Email: turco1987@ hotmail.com

(C) Los autores. Publicado por la Universidad Nacional Agraria La Molina.

This is an open access article under the CC BY

\section{INTRODUCCIÓN}

La inseminación artificial es una técnica que mejora genéticamente el rodeo. El control farmacológico del ciclo estral resulta de interés debido a que facilita la implementación de programas de inseminación artificial en los rodeos de cría, pudiendo inseminarse todos los animales en un tiempo prefijado (IATF), con la consiguiente mejora productiva (Uslenghi et al., 2016; Richardson et al., 2016). En las últimas décadas se han utilizado múltiples métodos de sincronización del estro para mejorar la eficiencia de los servicios de inseminación artificial (IA) y el manejo reproductivo del hato ganadero manteniendo una apropiada tasa de concepción.

"El control del ciclo estral mediante procedimientos biotécnicos tienen como base: el control del crecimiento y desarrollo folicular" (Lucy et al., 2004). "Crear una fase lútea de corta o larga duración" (Peralta et al., 2010). Y el control de la ovulación. Para tales fines se utiliza los esteroides (la Progesterona y /o Progestágenos), Prostaglandinas F-2_Alfa o sus análogos, GnRH o Benzoato de estradiol y el Cipionato de estradiol respectivamente (Bo et al., 2018).

"Estas técnicas han permitido ampliar el uso de la inseminación artificial e implementar en la ganadería de pequeños y medianos productores programas efectivos de mejoramiento genético" (Pedroso, 2003). "Como reducir las pérdidas económicas causadas por la ineficiente detección de celos" (Contreras, 2017). La eficiencia y calidad de los servicios de IA mediante el empleo de los diversos protocolos de IATF depende ante todo de la ocurrencia y el tiempo que transcurre entre el final del tratamiento con la progesterona y/o el progestágeno y la ocurrencia de la ovulación (Guilherme et al., 2020). "Y este evento depende del fármaco utilizado para inducir la ovulación" (Baruselli et al., 2004). Así como del estado fisiológico que tiene la hembra en reproducción al momento de efectuar el tratamiento. Las vacas en estado de anestro, la ocurrencia de la ovulación independiente del medicamento utilizado para la inducción del estro, se demora más la ocurrencia de la ovulación dado que la dinámica de crecimiento folicular es más lenta (Pedroso, 2003).

Investigaciones realizadas, con el fin de evaluar el uso del Benzoato de Estradiol o el Cipionato de Estradiol como inductores de la ovulación en la hembra bovina. Con este objetivo se ha experimentado administrar estos medicamentos en el momento de finalizado el tratamiento con progesterona o progestágenos o 24 horas más tarde. Los resultados obtenidos de fertilidad han variado entre 40 al $69 \%$ de fertilidad al primer celo (Cesaroni et al., 2007). "Pero, aún es escasa la información concerniente al uso de esto protocolos con 
ganado mestizo Bos indicus $x$ Bos taurus de grado de cruzamiento indeterminado bajo condiciones climáticas del trópico” (Lamb, 2012).

Estos antecedentes son la base de este trabajo, cuyo objetivo principal fue evaluar el impacto de un protocolo de IATF empleando Benzoato de Estradiol o Cipionato de Estradiol al momento o 24 horas más tarde de finalizado el tratamiento con progesterona en la eficiencia de los servicios de inseminación artificial a tiempo fijo, en vaquillas mestizas Bos indicus x Bos taurus criadas en pastoreo bajo las condiciones del trópico.

\section{MATERIALES Y MÉTODOS}

\section{Localización}

El estudio se realizó en Ecuador, Manabí, cantón Chone, parroquia convento. Encontrándose ubicado en las coordenadas geográficas S0 16 37,5 W80 02 12,3, con una altitud de 200 m.s.n.m. Condiciones climáticas se relacionan con una temperatura anual máxima $35,2^{\circ} \mathrm{C}$; media de $25,4^{\circ} \mathrm{C}$; y una mínima de $15,1^{\circ} \mathrm{C}$. En esta región existen dos épocas climáticas características del trópico seco muy bien distinguidas, la mayor fluctuación de lluvias comprende de enero a mayo, siendo la máxima precipitación de 250,00 mm/mes. Y otra época seca desde junio a noviembre durante los cuales la precipitación fluvial desciende casi a cero $\mathrm{mm} / \mathrm{mes}$, humedad relativa media de $86 \%$ (Sistema Nacional de la Pontificia Universidad Católica del Ecuador [SENAPUCE], 2011).

\section{Metodología de campo}

Se efectuó un experimento transversal mediante un diseño al azar completamente aleatorizado en campo. El período de duración de la investigación fue de 90 días. Para evaluar la eficiencia de los parámetros reproductivos de la investigación estuvieron sujetos a la metodología descrita por (Bulbarela, 2001).

\section{Animales}

Las vaquillas que se utilizaron para este experimento, se les realizó a todo un chequeo ginecológico del aparato genital por palpación rectal auxiliado por especulo vaginal. De este grupo fueron excluidas aquellas que tuvieron algún trastorno patológico en sus órganos genitales al examen clínico y solo o se seleccionaron las hembras con actividad cíclica. Para el desarrollo del experimento, se seleccionaron 72 vaquillas mestizas (Bos indicus x Bos taurus entre 28 a 32 meses de edad, con participación de los genotipos (Gyr, Brahmán, Holstein y Pardo Suizo). Estos animales tenían una condición corporal de 3,0 a 3,5 (Escala 1 al 5) y un peso entre 300 a 400 kilogramos.

\section{Estado sanitario manejo y alimentación}

Las vaquillas estaban bajo sistema de pastoreo extensivo con alimentación a base de pasto Saboya (Panicum máximum), suplementos minerales y agua a voluntad. El rebaño objeto de estudio estaba libre enfermedades infecto contagiosas (IBR/Brucelosis y Tuberculosis) según los registros y pesquisas periódicas de Agrocalidad.

\section{Tratamiento experimental}

Para la ejecución del experimento los animales fueron distribuidos en tres grupos A, B y C, según el tratamiento realizado:

A todos los animales el primer día (día cero), se les introdujo a las vaquillas un dispositivo intravaginal de silicona inerte impregnado con $0,5 \mathrm{mg}$ de progesterona natural (DIB® 0.5. Syntex). Además, se administró una inyección intramuscular 1,0 mg de Benzoato de Estradiol (Syntex); el día 8 se retiró el dispositivo intravaginal y se administró $500 \mu \mathrm{g}$ o 2 miligramo de un análogo de Prostaglandina -F2_Alfa (D+ Cloprostenol, Syntex). El tratamiento destinado para inducir la ovulación se efectuó según los grupos. (A, B o C) sometidos a los tratamientos:

Grupo A, $(n=24)$ : administración de (Cipionato de Estradiol, Syntex) $0,5 \mathrm{mg}$ el día cero al retirar el dispositivo intravaginal de progesterona. Grupo B, $(n=24)$ : administración de (Cipionato de Estradiol, Syntex) $1 \mathrm{mg}$ el día cero al retirar el dispositivo intravaginal de progesterona. Grupo C, $(n=24)$ : administración de $1 \mathrm{mg}$ de (Benzoato de Estradiol, Syntex) a las 24 horas de finalizado el tratamiento con progesterona.

Todas las vacas fueron inseminadas en tiempo fijo a las 56 a 58 horas de retirado el dispositivo intravaginal. Para efectuar la inseminación, se utilizó pajuelas de un mismo reproductor probado de raza girolando. El 
Vera, H., Pedroso, R., \& Hidalgo, G. (2021). Anales Científicos. 82(2), 198-205. DOI. 10.21704/ac.v82i2.1764

servicio fue realizado por un solo inseminador de más de ocho años de experiencia. El chequeo de gestación se realizó por palpación rectal 60 días después de la inseminación artificial.

\section{Análisis Estadísticos de los resultados}

Para la valoración de los datos experimentales se definieron las variables porcentajes de presentación de celo, concepción y preñez, comparados mediante prueba de comparación de proporciones y las diferencias entre medias según los tratamientos 0,5 mg (Cipionato de Estradiol), $1 \mathrm{mg}$ (Cipionato de Estradiol), y $1 \mathrm{mg}$ (Benzoato de Estradiol) utilizando la prueba Docima de Duncan. Para realizar este análisis se utilizó el Paquete Estadístico (Software y Soluciones de Analítica [SAS], 2001). El efecto del peso vivo en la eficiencia del tratamiento fue evaluado mediante un análisis de regresión logística, para realizar este análisis se utilizó el Software Estadístico InfoStat 2008 (Di Rienzo et al., 2008).

\section{RESULTADOS Y DISCUSIÓN}

En la (Tabla 1), se muestran los resultados de la presentación del celo en vaquillas tratadas con Cipionato de estradiol (ECP) y Benzoato de Estradiol (BE). Según estos datos las vacas tratadas con $1 \mathrm{mg}$ de ECP mostraron un mayor porcentaje de vacas en celo $(\mathrm{p}<0,05)$ respecto al grupo A y B respectivamente. De acuerdo con estos datos, el grupo $\mathrm{A}$ mostró una mayor tasa de concepción y preñez $68,10 \%$ y $62,5 \%$. Estos valores fueron más altos $(p<0,05)$, que los hallados en los grupos $\mathrm{B}$ y $\mathrm{C}$ respectivamente con los otros grupos experimentales.

En la (Tabla 2), se señala la distribución del celo en las vaquillas en los diferentes grupos A, B y C. De acuerdo con estos hallazgos en el grupo (A), la mayoría de las vaquillas presentaron el estro entre 36 a 48 horas de finalizado el tratamiento con progesterona administración de Prostaglandina F-2-Alfa y el ECP, siendo inseminadas 8 a 20 horas más tarde. En el grupo (B), la distribución del celo fue uniforme durante las 56 horas posteriores al tratamiento. Así, el $50 \%$ de los animales, presentaron celo y fueron inseminados 20 a 32 horas más tarde y el otro $50 \%$ a las 56 horas de finalizado el tratamiento de sincronización. Con respecto al grupo (C), en este grupo de vacas, el 50\% de los celos fueron detectados entre 24 a 48 y el $50 \%$ restante a las 56 horas de realizado el tratamiento de sincronización. En este sentido, la mitad de los animales fueron inseminados entre 8 a 32 horas de ser observadas en celo y el $50 \%$ al momento de ser detectado en estro.

En la presente investigación el porcentaje de presentación de celo fue similar a los informados por Uslenshi et al. (2016). Esto sugiere, que estos protocolos pueden ser utilizados con una adecuada respuesta de los animales a la presentación del estro.

En este sentido, se han introducido nuevos sistemas de producción, que contribuyen significativamente al aumento de la productividad del ganado. Pero, la continuidad del incremento de la productividad en el futuro exige obligatoriamente, el inicio y la conducción ininterrumpida de los trabajos de mejoramiento genéticos, que consisten en la selección del ganado en base a sus características hereditarias (Thatcher, 2005).

Tabla 1. Efecto de tratamientos con ECP y BE sobre porcentaje de celo, concepción y preñez en vaquillas mestizas Bos indicus x Bos taurus criadas en clima tropical.

\begin{tabular}{|c|c|c|c|c|c|c|}
\hline Tratamientos & Grupos & $\mathbf{N}$ & $\begin{array}{c}\text { Número de animales } \\
\text { en estro }\end{array}$ & $\begin{array}{c}\text { Porcentaje de vacas } \\
\text { en celo }\end{array}$ & Concepción (\%) & $\begin{array}{c}\text { Preñez } \\
(\%)\end{array}$ \\
\hline ECP-0.5 mg & $\mathrm{A}$ & 24 & 22 & $91,60^{\mathrm{a}}$ & $68,10^{\mathrm{a}}$ & $62,50^{a}$ \\
\hline $\mathrm{ECP}-1 \mathrm{mg}$ & B & 24 & 24 & $100.00^{b}$ & $50,0^{\mathrm{b}}$ & $50,0^{b}$ \\
\hline $\mathrm{BE}-1 \mathrm{mg}$ & $\mathrm{C}$ & 24 & 22 & $91,60^{\mathrm{a}}$ & $59,0^{\mathrm{b}}$ & $54,10^{\mathrm{b}}$ \\
\hline Totales & & 72 & 68 & 0,0 & 58,80 & 55,50 \\
\hline
\end{tabular}

Letras diferentes por columnas difieren entre sí $\mathrm{p}<0,05$ 


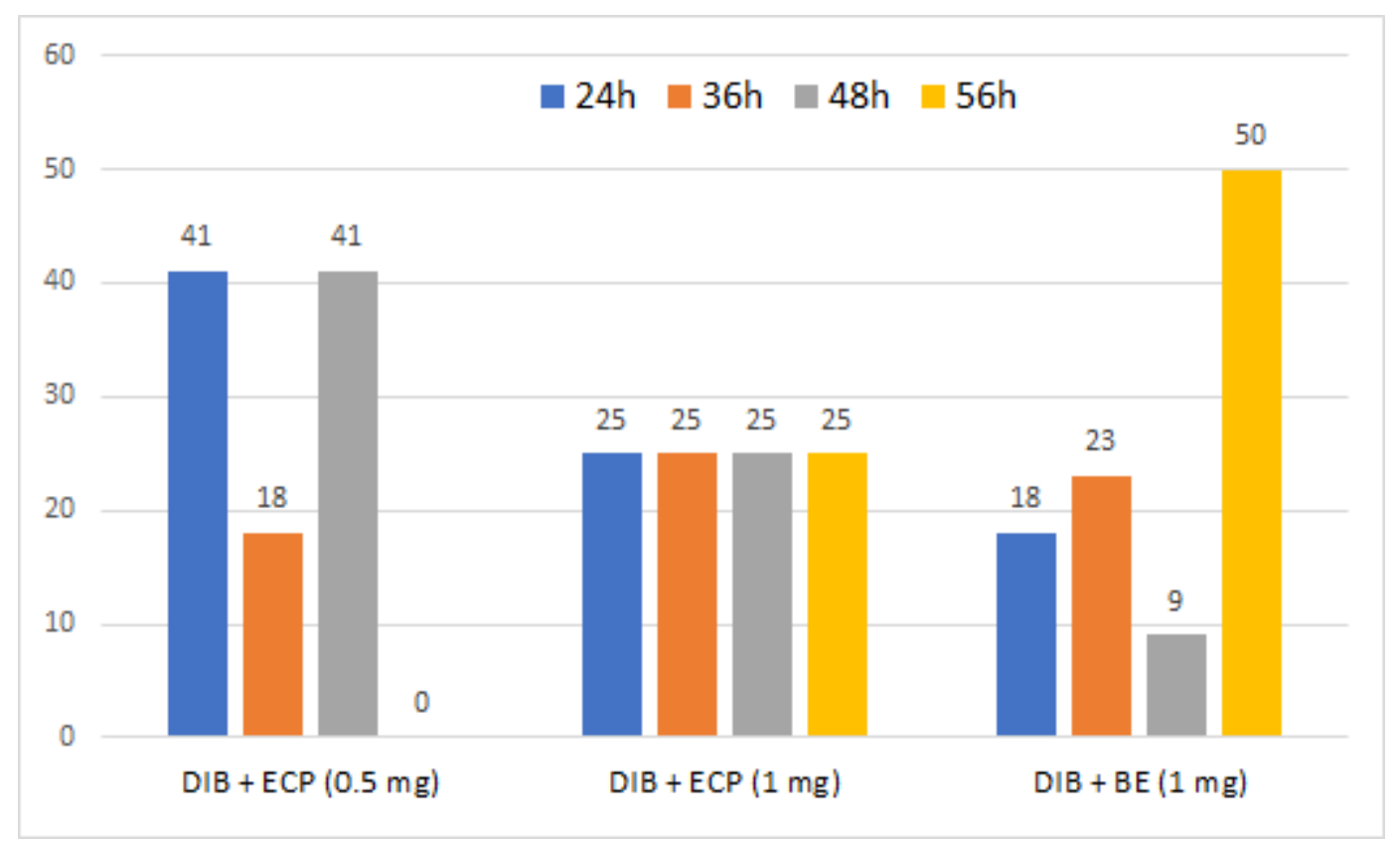

Figura 1. Frecuencia de estros de vacas tratadas con tres protocolos de sincronización de celo

Según el análisis estadístico efectuado con el fin de demostrar el efecto del peso vivo de las vaquillas que tenían entre 300 a 400 kilos no influenciaron en la eficiencia de los servicios de inseminación artificial de los grupos experimentales, mostrando resultados no significativos $(\mathrm{p}<0,05)$ de error.

"En este contexto, la aplicación de la tecnología de inseminación artificial juega un papel prioritario por ser la herramienta técnica de mayor eficiencia para introducir la mejora genética" (Marizancén y Artunduaga, 2017).

Al respecto, uno de los factores que limita su introducción en los rebaños de pequeños y medianos productores en el trópico, es la baja eficiencia en la detección del estro y la realización en el tiempo óptimo de los servicios de inseminación artificial (Pedroso, 2003). "Por consiguiente, en las últimas décadas se han desarrollado múltiples esfuerzos por desarrollar procedimientos biotécnicos, que permitan sincronizar el celo, la ovulación con el objetivo de realizar la inseminación en tiempo fijo garantizando una adecuada fertilidad" (Martins et al., 2017).

"Con el fin de inducir la ovulación posterior a la ocurrencia de una fase lútea de corta o larga duración ha sido practicada con varios productos farmacéuticos en los últimos 10 años" (Hockey et al., 2010). Así, la aplicación de estradiol (ECP o BE), al inicio y al final de un tratamiento con progesterona inducir la ovulación e implementar una inseminación artificial en tiempo fijo con adecuada tasa de preñez ha sido probado en múltiples estudios donde se han obtenidos buenos resultados de fertilidad (Bo et al., 2018).

Para lograr estos objetivos es necesario ejercer un control del crecimiento y emergencia de las olas foliculares. Con esta finalidad, al inicio del tratamiento, su combinación la administración de estrógeno con la progesterona y este hecho producen en primer lugar la atresia del folículo dominante y el surgimiento de una nueva ola folicular sincronizada, tres a cuatro días más tarde. Una vez retirado el dispositivo de progesterona, la aplicación de una dosis adecuada de estradiol puede inducir la descarga de LH lográndose una ovulación sincronizada 48 a 64 horas más tarde (Colazo et al., 2004) con una fertilidad aceptable.

Uno de los aspectos de mayor interés en estos resultados es lo referente a la distribución de la observación de la manifestación del estro verdadero, en los animales tratados posterior al tratamiento. De acuerdo con los datos, aportados en el presente estudio, la manifestación del celo en las vacas de los grupos (B y C) tuvo una mayor dispersión. Mientras, que en el 
grupo A se observó una mayor concentración de los celos entre 36 y 48 horas de finalizado el tratamiento con progesterona. Este resultado confirma las observaciones de Uslenshi et al. (2016), quienes afirman que ECP en dosis de $0,5 \mathrm{mg}$ administrado posterior a un tratamiento con progesterona de ocho días permite una menor dispersión de la presentación de los estros y las ovulaciones en vacas sin cría al pie con una fertilidad entre $47 \%$ al $60 \%$.

Según lo expuesto por Macmillan (2010), el estradiol posterior al tratamiento con progesterona resulta conveniente aplicarlo a las 24 horas de haber retirado el dispositivo de progesterona periodo en que generalmente ha desaparecido la progesterona suplementada en la sangre y no a las cero horas cuando podría haber progesterona circulando, en caso que exista un cuerpo lúteo en fase de regresión por el tratamiento con Prostaglandina F-2-Alfa. En este caso, la administración de ECP puede provocar la atresia del folículo dominante y el surgimiento de una nueva ola folicular sincronizada o forzar la ovulación de un folículo todavía no maduro.

Similares observaciones fueron realizadas por Bosolasco et al. (2021), quienes señalaron, que aplicando el estradiol a las cero horas ocurre una mayor dispersión en el momento de la ovulación con respecto a la administración de este fármaco a las 24 horas. Pero, Ross et al. (2002), "no encontró diferencias en el momento de la ovulación cuando se administra el estradiol a las cero o 24 horas de finalizado el tratamiento con progesterona"

La aplicación de estradiol, en sus diferentes sales y dosis, ya sea al principio o al final de un tratamiento con progesterona para sincronizar celo y la ovulación, ha sido objeto de numerosos estudios. Con respecto, a la aplicación de ECP al finalizar un tratamiento con progesterona fue evaluado en varios experimentos (Cesaroni et al., 2007; Sa Filho et al., 2011) con el fin de mejorar la fertilidad y evitar un cuarto movimiento de las hembras tratadas. "Al respecto, la tasa de concepción y preñez obtenida en esta investigación se encuentra dentro del rango de los resultados informados anteriormente por" (Butller et al., 2011).

Acorde con los hallazgos experimentales de la presente investigación, la tasa de fertilidad fue superior $(p<0,05)$ en las vacas tratadas con $0,5 \mathrm{mg}$ de ECP a las cero horas de finalizado el tratamiento con progesterona comparado con los demás grupos tratados (B) y C respectivamente. Esto indica, que es posible mejorar la tasa de fertilidad y evitar un nuevo movimiento del rebaño mediante la aplicación de ECP a la cero hora de finalizado el tratamiento con progesterona. Este resultado permite sustentar la hipótesis del presente trabajo de investigación, y son comparables con los informados previamente en los estudios de (Ferrerira et al., 2015).

Referente al efecto del peso corporal de las vaquillas al inicio del experimento en la eficiencia de los servicios de IATF en la presente investigación, no se pudo comprobar que este factor influyera significativamente con los hallazgos obtenido. Pero, esta información se corresponde con los resultados expuesto por Hidalgo et al. (2012), quienes señalaron, que la tasa de preñez no dependió del peso corporal en vaquillas mestizas $(\mathrm{P}=0,09)$ no obstante manifestó que el peso corporal de una vaquilla para que sea servida por primera vez debería tener 340 kilos promedio para el trópico seco criadas en condiciones extensivas de pastoreo. Además, estos datos se contrastan a los encontrados por López et al. (2011) que las hembras Brahmán completaron su desarrollo genital cuando alcanzan peso promedio de $363 \mathrm{~kg}$ para comenzar a ciclar, criadas en condiciones considerables de alimentación en pastoreo y suplementación energética y proteica.

\section{CONCLUSIONES}

De acuerdo con los resultados obtenidos se concluye que administrar ECP en dosis de $0,5 \mathrm{mg}$, aplicadas a las cero horas de finalizado el tratamiento con progesterona mostró la mejor uniformidad en la manifestación del celo y, tuvo un mayor porcentaje de concepción y preñez al ser comparado con la administración de ECP en dosis de $1 \mathrm{mg}$. o el BE. Como también el uso del ECP en los protocolos de sincronización del celo a tiempo fijo administrado el mismo día de finalizado el tratamiento con progesterona permite disminuir un día en las labores de campo con adecuadas tasas de preñez en vaquillas mestizas Bos indicus x Bos taurus criadas en pastoreo bajo las condiciones climáticas del trópico. 


\section{Conflictos de intereses}

Los autores firmantes del presente trabajo de investigación declaran no tener ningún potencial conflicto de interés personal o económico con otras personas $\mathrm{u}$ organizaciones que puedan influir indebidamente con el presente manuscrito.

\section{Contribuciones de los autores}

Preparación y ejecución: HVA; RPS; Desarrollo de la metodología: HVA; GHB; Concepción y diseño: GHB; RPS; Edición del artículo: GHB; Supervisión del estudio: HVA; RPS

\section{REFERENCIAS}

- Baruselli, P. S., Reirs, E. L., Marques, M. O., Nasser, L. F., 6 Bo, G. A. (2004). The use of hormonal treatments to improve reproductive performance of anestrous beef cattle in tropical climates. Anim Reprod. Sci. 82-83, 479-486. https://doi.org/10.1016/j.anireprosci.2004.04.025

- Bó, G., Huguenine E., de la Mata JJ., Núñez, OR., Baruselli PS., \& Menchaca A. (2018). Programs for fixed-time artificial insemination in South American beef cattle. Proceedings of the 10th International Ruminant Reproduction Symposium (IRRS) Foz do Iguaçu, PR, Brazil, September 16th to 20th.

- Bosolasco, D., Nuñez, R., de Brun, O. V., Meikle, A., \& Menchaca, A. (2021). Estradiol cypionate administered at the end of a progesterone-based protocol for FTAI induces ovulation and improves postovulatory luteal function and uterine environment in anestrous beef cows. Theriogenology, 162, 74-83. ISSN 0093-691X https://doi.org/10.1016/j.theriogenology.2021.01. 003.

- Bulbarela, G.G. (2001). Comportamiento reproductivo de un hato Holstein en clima semicalidos. [Tesis de licenciatura]. Facultad de Medicina Veterinaria y Zootecnia. Universidad Veracruzana. Veracruz, México.

- Butler, H. M., Butler, A., Etcheverry, E., \& Cesaroni, G. C. (2011). Efecto de la dosis de cipionato de estradiol al finalizar un tratamiento con progesterona sobre el porcentaje de preñez a la IATF en vaquillonas. Rev. Taurus, 13(52), 40-45.

- Cesaroni, G., Butler, H. M., \& Durand, M. J.
(2007). Evaluación del uso de dos ésteres de estradiol sobre la tasa de fertilidad a la IATF en vacas secas, tratadas con un dispositivo intravaginal con progesterona. Rev Taurus, 9, 1218.

- Colazo, M. G., Kastelic, J. P., Martinez, M. E., Whittaker, P. R., Wilde, R., Ambrose, J. D., Corbett, R., \& Mapletoft, R. J. (2004). Fertility following fixed timed AI in CIDR-treated beef heifers given $\mathrm{GnRH}$ or estradiol cypionate and feed diets supplemented with flax seed or sunflower seed. Theriogenology, 61, 1115-24. 11.

- Contreras, L. A. (2017). Inseminación Artificial en Bovinos: Problemáticas y Actualidad. Revista electrónica: Engormix. Recuperado Junio 2020, de: https://www.engormix.com/ganaderialeche/articulos/inseminacion-artificialbovinosproblematicas-t40323.htm

- Di Rienzo, J. A., Casanoves, F., Balzarini, M. G., Gonzalez, L., Tablada M. \& Robledo, C. W. (2008). InfoStat, versión 2008, Grupo InfoStat, FCA, Universidad Nacional de Córdoba, Argentina.

- Duncan, D. B. (1955). Multiple range and multiple $\mathrm{F}$ tests. Biometrics, 11, 1-42.

- Ferreira, R. M., Ayres H., Gimenes, L. U., Torres, F. P., Lima, L. A., Veras, M. B., Guida, T. G., Sala, R. V., \& Baruselli, P. S. (2015). La ovulación inducida con cipionato de estradiol permite flexibilidad en el momento de la inseminación y elimina la necesidad de la hormona liberadora de gonadotropina en los protocolos de IA cronometrados para las vacas lecheras. Reproducción fertilidad y desarrollo, 29(3), 468475. https://doi.org/10.1071/RD15270

- Guilherme, M., Motta, J., Drum, J., Consentini, C., Prata, A., Monteiro, P., Melo, L., Alvarenga, A., Wiltbank, M., \& Sartori, R. (2020). Progesteronebased timed AI protocols for Bos indicus cattle I: Evaluation of ovarian function. Theriogenology, 145 , 126-137. https://doi.org/10.1016/j.theriogenology.2020.01. 030

- Hidalgo, G. A., Zambrano, A. E., \& Macías, J. I. (2012). Influencia de los órganos reproductores sobre la fertilidad en vaquillonas mestizas inseminadas a tiempo fijo. Revista EspamCiencia, 3(2), 119-126. 
- Hockey, C. D., Morton, J. M., Norman, S.T., \& McGowan M.R. (2010). Una mejor predicción del tiempo de ovulación puede aumentar las tasas de embarazo a la inseminación artificial en ganado lechero lactante. Reprod. Domestico Anim, 45(6), 239-248. https://doi.org/10.1111/j.14390531.2009.01548.x

- Lamb, G. (2012). Methods to increase reproductive efficiency in cattle. Thesis $\mathrm{PhD}$. United States of Agriculture. University of Florida. pp. 100.

- López, S., Robson, C., \& Menchón, P. (2011). Estudio de la precocidad sexual de vaquillas en cabañas de la raza Brahman. Su potencial utilización en programas de mejora genética. Estación Experimental del INTA Mercedes. Asociación de Criadores de Brahman Argentina. Serie Técnica No 52. ISSN 0327-3075. pp 12-13.

- Lucy, M. C., McDougall S., \& Nation, D. P. (2004). The use of hormonal treatments to improve the reproductive performance of lactating dairy cows in feedlot or pasture-based management systems. Anim Reprod Sci, 82-83, 495-512. https://doi.org/10.1016/j.anireprosci.2004.05.004

- Macmillan, K. L. (2010). Recent advances in the synchronization of estrus and ovulation in dairy cows. J. Reprod. Dev, 56:S, 42-S47.

- Marizancén, M. A., \& Artunduaga, P. L. (2017). Mejoramiento genético en bovinos a través de la inseminación artificial y la inseminación artificial a tiempo fijo. Revista de Investigación Agraria y Ambiental, 8(2), 247-259. ISSN 2145-6097 https://hemeroteca.unad.edu.co/index.php/riaa/arti cle/view/2050/2301

- Martins, T., Talamoni, J. P. Sponchiado, M., Maio, J. R. G., Nogueira, G. P., Pugliesi, G., \& Binelli, M. (2017). Impact of estradiol cypionate prior to TAI and progesterone supplementation at initial diestrus on ovarian and fertility responses in beef cows. Theriogenology, 104, 156-163, ISSN 0093$691 \mathrm{X}$

Doi: https://doi.org/10.1016/j.theriogenology.2017.08. 017.

- Pedroso, R. (2003). Métodos Biotécnico para mejorar la eficiencia de los servicios de inseminación artificial y los programas de inducción y sincronización del celo. [Tesis, Dr.
Cs.] Julio 17, 2003. UNAH. Ciudad Habana Cuba.

- Peralta, J. A., Aké, J. R., Centurión, F. G., \& Magaña, J.G. (2010). Comparison of estradiol cypionate and estradiol benzoate effects on ovaric activity, estrus and ovulation on anestrus Bos indicus cows. J Anim Vet Adv., 9(3), 466-470.

- Richardson, B. N., Hill, S. L., Stevenson, J. S., Djira, G. D., \& Perry, G. A. (2016). Expression of estrus before fixed-time AI affects conception rates and factors that impact expression of estrus and the repeatability of expression of estrus in sequential breeding seasons. Anim Reprod Sci, 166, 133-140. https://doi.org/10.1016/j.anireprosci.2016.01.013

- Ross, J. P. (2002). Sincronización del celo y de la ovulación en vacas con cría al pié mediante el uso de acetato de medroxiprogesterona y benzoato de estradiol. [Tesis MSc Universidad Nacional].

- Sá Filho, M. F., Santos J. P., Ferreira R. M., Sales, J. N. S., \& Baruselli, P. S. (2011). Importance of estrus on pregnancy per insemination in suckled Bos indicus cows submitted to estradiol/progesterone-based timed insemination protocols. Theriogenology, 76(3), 455-463. https://doi.org/10.1016/j.theriogenology.2011.02. 022

- SAS (2001). System for Elementary Statistical Analysis, Second Edition. EUA.

- SENAPUCE: Sistema Nacional de la Pontificia Universidad Católica del Ecuador. 2011. Condiciones climáticas del cantón Chone. Estación Meteorológica SENAPUCE, comunidad Garrapata, Chone-Manabí- Ecuador.

- Thatcher, W. W. (2005). Manejo Reproductivo del Bos Taurus y Bos indicus. Simposio. Primer Congreso Internacional de Producción Animal. Palacio de la Convenciones. Ciudad Habana, Cuba, 7-11 Noviembre 2005.

- Uslenghi G., Cabodevila, J., \& Callejas, SS. (2016). Effect of estradiol cypionate and GnRH on synchronization of ovulation and pregnancy rate at FTAI in non-suckled cows. InVet, 18(2), 301-306. ISSN 1668-3498 (en línea). http://www.fvet.uba.ar/archivos/publicaciones/inv et/vol18-2-2016/Vol_182_2016_ARTICULO_01.pdf 